\title{
El subsuelo de lo político: Sustratos sociales, culturales, comunicacionales y tecnológicos de la acción colectiva en América Latina en el siglo XXI
}

O subsolo do político: substratos sociais, culturais, comunicacionais e tecnológicos da ação coletiva na América Latina do século XXI

The Subsoil of the Political: Social, Cultural, Communicational, and Technological Substrates of Collective Action in Latin America in the XXI century

\author{
José Antonio VILLARREAL VELÁSQUEZ \\ Ecuador \\ Universidad de Bielefeld-Alemania \\ jose.villarreal@uni-bielefeld.de \\ ORCid 0000-0002-0102-400X \\ Gilberto RESCHER \\ Alemania \\ Universidad de Hamburgo-Alemania \\ gilberto.rescher@uni-hamburg.de \\ ORCid oooo-0oo2-1121-4691
}

Chasqui. Revista Latinoamericana de Comunicación

$N .^{o}$ 148, diciembre 2021 marzo 2022 (Sección Monográfico, pp. 17-30)

ISSN 1390-1079 / e-ISSN 1390-924X

Ecuador: CIESPAL

Recibido: 20-11-2021 / Aprobado: 13-12-2021 


\title{
Resumen
}

Con este dossier nos proponemos contribuir a las discusiones actuales sobre la configuración de lo político en América Latina. Buscamos discutir, desde las ciencias sociales, aquellos "enigmas" relacionados a las lógicas culturales, sociales, comunicacionales y tecnológicas del acontecer político y su fundamento en los respectivos sistemas de saber enraizados en los muy diversos actores que son partícipes de los procesos políticos contemporáneos.

Nos inspiramos en la noción el subsuelo político desarrollada por el filósofo boliviano Luis Tapia (2008) y, haciendo una pequeña variación, proponemos el subsuelo de lo político como una categoría de análisis, y no como un objeto de estudio. Como una categoría de análisis histórica, social y política, el subsuelo de lo político sirve para entender, desde una perspectiva transdisciplinaria y multidimensional, las diversas formas de interacción a través de las cuales se (de)construyen las fronteras entre lo social y la política.

Desde el subsuelo de lo político pretendemos comprender cómo los elementos culturales, comunicacionales, espaciales y tecnológicos fluyen, se articulan (bajo distintas formas y escalas), adquieren sentido e integran un sustrato nunca fijo- del que se alimentan los procesos y las dinámicas de movilización social y societal. Estas dinámicas, impulsadas por grupos, movimientos y actores muchas veces representados como marginales o minoritarios, pero presentes durante las dos últimas décadas en la región latinoamericana, son el centro de atención de este dossier.

Palabras clave: acción colectiva, lo político, tecnología digitales, redes sociales

\begin{abstract}
The special issue attempts to contribute to the current discussions on the configuration of the political in Latin America. From the social sciences, we seek to discuss those "enigmas" related to the cultural, social, communicational, and technological logics of political events, as well as to the systems of knowledge rooted in those actors that participate in contemporary political processes.

Drawing inspiration from Bolivian philosopher Luis Tapia's (2008) notion political subsoil, and making a slight variation of it, the special issue develops the category the subsoil of the political. We use the subsoil of the political as a category of analysis, and not as an object of study. As a category of historical, social and political analysis, the subsoil of the political serves to understand, from a transdisciplinary and multidimensional perspective, the various forms of interaction through which the boundaries between the social and the political are (de)constructed.

From the subsoil of the political, we seek to understand how cultural, communicational, spatial and technological elements flow, articulate (under different forms and scales) and acquire meaning, and integrate a substrate never fixed. From this substrate, groups, movements and actors sometimes represented as marginal or minority, but politically meaningful during the last
\end{abstract}


two decades in the Latin American region, trigger processes and dynamics of social and societal mobilization, which are the focus of this dossier.

Keywords: Collective action, politics, digital technology, social networks

\section{Resumo}

Com este dossiê pretendemos contribuir para as discussões atuais sobre a configuração da política na América Latina. Procuramos discutir, a partir das ciências sociais, aqueles "enigmas" relacionados com a lógica cultural, social, comunicacional e tecnológica dos eventos políticos e seus fundamentos nos respectivos sistemas de conhecimento enraizados nos mais diversos atores que participam dos processos políticos contemporâneos.

Nós inspiramos pela noção de subsolo político desenvolvida pelo filósofo boliviano Luis Tapia (2008) e, fazendo uma pequena variação, propomos $o$ subsolo do político. Utilizamos o subsolo do político como categoria de análise, e não como objeto de estudo. Como categoria de análise histórica, social e política, o subsolo do político serve para compreender, de uma perspectiva transdisciplinar e multidimensional, as várias formas de interação através das quais as fronteiras entre o social e o político são (de)construídas.

A partir do subsolo do político, procuramos entender como os elementos culturais, comunicacionais, espaciais e tecnológicos fluem, articulam (sob diferentes formas e escalas), adquirem significado e integram um substrato nunca fixo- do qual se alimentam os processos e dinâmicas de mobilização social e societal. Esses processos, impulsionados por grupos, movimentos e atores muitas vezes representados como marginais ou minoritários, embora presentes durante as duas últimas décadas na região latino-americana, são o centro de atenção do nosso dossiê.

Palavras-chave: ação coletiva, política, tecnologia digital, redes sociais

La economía, la cultura, la comunicación, la religión, en general lo social y, sobre todo, la política (entendida esta como un conjunto de prácticas, formas de pensar e instituciones que regulan y organizan la convivencia humana) han experimentado una variedad de cambios, transiciones y transformaciones que se han acelerado durante las dos primeras décadas del siglo XXI. Lo político, el conflicto, el antagonismo y el desacuerdo, según los supuestos del pensamiento filosófico posfundacional, se han destacado por ser elementos aceleradores. Mejor aún, lo político es comprendido desde esta perspectiva como un momento instituyente de dichas transformaciones. Su principal característica es el ser inasible y contingente. Lo político se manifiesta como una dimensión ontológica de la política.

No obstante, como tratamos de reflexionar en este dossier, en diálogo con esta visión posfundacional, lo político implica también situaciones de negociación, colaboración y acuerdo. Más aún, lo político aparece, se constituye, 
toma forma y se nutre de un conjunto de interacciones, formas de hacer y decir que están arraigados en un sinnúmero de lógicas y racionalidades políticas; por lo tanto, precisa de un análisis más complejo. Por debajo de conflictos, acuerdos y desacuerdos se configura un subsuelo en el que se depositan una diversidad de elementos culturales, sociales, comunicacionales y tecnológicos cuyos significados y usos prácticos son pertinentes e imprescindibles de estudiar para desarrollar una comprensión teórico-metodológica más amplia sobre la especificidad de lo político en las sociedades latinoamericanas en el siglo XXI.

Con este dossier, nos proponemos contribuir a las discusiones actuales sobre la configuración de lo político en América Latina. Buscamos discutir, desde las ciencias sociales, aquellos "enigmas" relacionados a las lógicas culturales, sociales, comunicacionales y tecnológicas del acontecer político y su fundamento en los respectivos sistemas de saber enraizados en los muy diversos actores que participan en los procesos políticos contemporáneos.

Consideramos que es necesario comprender con mayor profundidad analítica las raíces sociales de los cuestionamientos, los (des)acuerdos y las controversias que han (des)estabilizado las fronteras entre sociedad y política y han sido protagonizadas por una amplia gama de actores, grupos y movimientos sociopolíticos. Actores, grupos y movimientos cuyas dinámicas de acción colectiva y contenciosa - no siempre visible y muchas veces silenciosa- han puesto en escena el uso de tradicionales y nuevas formas de organización, el establecimiento de relaciones de subordinación y sublevación dinámicas, la creación de lenguajes, signos, símbolos y técnicas dramatúrgicas, los artes del disfraz político, la escenificación estratégica y el arte de no ser gobernado, parafraseando a James Scott (1990; 2009). A esto se suma, el uso de las redes sociales (Castells, 1996; 2012; Bringel y Pleyers, 2017), tecnologías digitales o tecnopolítica (Torret, 2013) y, aún más importante, la (des)politización de los tiempos y los no-lugares de la política (Tapia, 2008). En resumen, nos interesan aquellas prácticas, discursos y espacios desde donde y a través de los cuales las dinámicas políticas se (in)visibilizan, (re)inventan y robustecen.

Además, el dossier está motivado por la necesidad de encontrar nuevos caminos teóricos y pistas empíricas útiles para la construcción o ampliación de una teoría de alcance medio que conjugue exploraciones de los diversos niveles en los cuales los procesos sociopolíticos estudiados toman lugar. Nuestra idea es abrir el enfoque investigativo hacia aquellos espacios, grupos y procesos que frecuentemente pasan desapercibidos por "carecer de importancia", pero que, justamente por aquello, poseen un potencial analítico para comprender los entrelazamientos entre los diversos niveles (desde lo local hasta lo global) presentes en las interacciones políticas. Así, con este dossier pretendemos establecer los primeros pasos hacia la formulación de una perspectiva analítica y crítica que sea útil para estudiar diversos actores y contextos en el "sur global”,

1 Esta perspectiva analítica no es solamente relevante y factible para pensar lo político en sociedades latinoamericanas. Existen otros trabajos que comparten intereses e inquietudes similares como, por ejemplo, 
pero que, a la vez, abra caminos para pensar y comparar los procesos políticos y la acción colectiva que ha emergido en el "norte global", ahí donde supuestamente los sistemas políticos son más funcionales e institucionalizados.

Algunas preguntas que orientan la discusión de este dossier son: ¿Quéformas de resistencia, subordinación y sublevación se entretejen en el subsuelo político? ¿Qué relevancia teórica tiene el carácter no-formalizado y no-institucional del subsuelo político? ¿Cómo interactúan actores con posturas y convicciones políticas muy diversas en estos espacios y, en términos más generales, cómo influye la posición social de los actores que se mueven en estos ámbitos y que en el caso de grupos a quienes se les asigna socialmente una posición marginalizada o de minoría carecen de cierta "visibilidad"? ¿Qué tipo de transformaciones tecnológicas y cuáles son los usos de las nuevas herramientas de comunicación digital qué caracterizan a las dinámicas de acción colectiva actuales? ¿Cuáles son los nuevos métodos de análisis y los usos de metodologías para la producción de conocimientos de los fenómenos sociopolíticos en América Latina?

\section{El subsuelo de lo político como categoría de análisis}

Las y los lectores podrán reconocer en el título del dossier que nos inspiramos en la noción el subsuelo político desarrollada por el filósofo boliviano Luis Tapia (2008). . Haciendo una pequeña variación de la misma, preferimos usar: el subsuelo de lo político. Esta variación implica entender el subsuelo de lo político como categoría de análisis, y no como un objeto de estudio. Como una categoría de análisis histórica, social y política sirve para entender, desde una perspectiva transdisciplinaria y multidimensional, las diversas formas de interacción a través de las cuales se (de)construyen las fronteras entre lo social y la política.

De esta manera, el subsuelo de lo político reorienta los estudios sobre lo político que, bajo la influencia del mencionado canon del pensamiento filosófico posfundacional, ha sido definido en contraposición a la política, como un momento - disruptivo y espontáneo- de institución de lo social. ${ }^{3}$ De igual forma, la categoría el subsuelo de lo político busca ampliar los estudios de la

\footnotetext{
Nageeb (2007) y Lachenmann/Dannecker (2008) que analizan las actividades políticas feministas en países islámicos. Büschges y Pfaff (2003) que realizan una comparación de la etnización de la política en el sur de Asia y la región Andina. Además, desde una visión más amplia, están los casos recopilados por los estudios de Vincent (2002) y Nugent y Vincent (2004). Todos estos trabajos coinciden en una concepción de lo político más amplia y toman en cuenta a lugares de la "no-política" y a actores que frecuentemente de cierta forma son vistos como pasivos y sumisos y como no aptos para la política. Para una discusión sobre cómo tales perspectivas han influido en el desarrollo de los acercamientos de la sociología alemana, por ejemplo, hacia el "sur global" véase también Gerharz y Rescher (2021).

2 El subsuelo político, dice Tapia, es aquel conjunto de prácticas y discursos políticos que no son reconocidos social y estatalmente, pero emergen como forma de acción, interacción y opinión sobre la dimensión política y de gobierno de las sociedades. El subsuelo político contiene crítica, desdén, ironía, sabotaje, parodia, en algunos casos formas alternas y alternativas. En el seno del subsuelo político se generan sus peculiares formas de solidaridad y comunicación (2008, p. 97).

3 Para una reflexión más profunda sobre las diferencias entre la política y lo político ver Chantal Mouffe (2007) y Olivert Marchart (2009).
} 
ciencia política que reducen lo político a una cuestión normativa y lo localizan en el ámbito institucional/estatal, sin percibir cómo el sistema político se extiende y se basa en ámbitos societales más amplios.

A pesar de que el conflicto, la irrupción y la contingencia son elementos integrales de la reflexión sobre lo político, la categoría que proponemos busca explorar más allá de las potencialidades inscritas en las manifestaciones disruptivas del conflicto y la reducción de la complejidad que caracteriza al enfoque institucional sobre la política. Desde el subsuelo de lo político pretendemos entender también cómo los elementos culturales, comunicacionales, espaciales y tecnológicos fluyen, se articulan (bajo distintas formas y escalas), adquieren sentido e integran un sustrato — nunca fijo- del que se alimentan los procesos y las dinámicas de movilización social y societal. Dichas dinámicas han sido impulsadas por grupos, movimientos y actores muchas veces representados como marginales o minoritarios, pero que han estado presentes en distintos contextos y escenarios durante las dos últimas décadas en la región latinoamericana. Esto, como señala Rescher, se debe a que frecuentemente la persistencia de lógicas políticas fomenta una considerable estabilidad del sistema que facilita la continua invisibilización del subsuelo político ignorando a gran parte de los múltiples actores allí presentes, sobre todo, si se trata de grupos considerados como marginales o minoritarios (Rescher 2018a).

La importancia de esta perspectiva deriva del hecho de que este subsuelo de lo político se encuentra "bajo" la esfera política visible con sus manifestaciones formalizadas e institucionales. Es un ámbito político que regularmente no se observa como tal, ya que no cumple con los requisitos de los conceptos formales (o formalistas) de política o incluso los contradice. El subsuelo de lo político comprende espacios de informalidad, flexibilidad e incluso una especie de caos que está presente en la cotidianidad y los mundos de vida de muchos actores sociales que no son concebidos como políticos.

Desde el subsuelo de lo político indagamos cómo y dónde los actores definen y posicionan temas controversiales, problematizan relaciones y estructuras sociales, crean vínculos y (des)politizan espacios, herramientas tecnológicas, experiencias, territorios y lugares considerados como no tradicionales para hacer política y desde los cuales los límites que separan sociedad y política son (socialmente) construidos, se tornan borrosos o se mantienen estables. ${ }^{4}$

Esta categoría pretende generar nuevas y valiosas perspectivas al estudiar, por ejemplo, las relaciones entre procesos políticos que acontecen en diversos niveles; las interdependencias entre las formas de organización, los procesos comunicacionales y de diálogo que los actores activan; las prácticas de participación política (ciudadana y electoral), las formas en que diversas racionalidades políticas están arraigadas a múltiples sistemas de saber que se

4 Con relación a la construcción discursiva de las separaciones entre distintas esferas sociales, a partir de una lógica moderna y eurocéntrica, ver la discusión que desarrolla Bruno Latour (2007). 
nutren de mundos y experiencias cotidianas de los actores, así como también desde sus relaciones con las dimensiones espacio-temporales en las que se configuran. Aquí se destaca, entre otras, la confrontación, cambio y persistencia de dinámicas opuestas: aquella que fomenta la estabilidad del sistema y aquella que promueve su cambio. Por ello, nuestro foco de interés incluye a aquellos actores políticos que resisten, cuestionan y se oponen al status quo, así como también a aquellos que lo reproducen, legitiman y lo promueven, sin perder de vista que existen procesos contradictorios e incluso entrelazados que apuntan al mismo tiempo a ambas direcciones.

Lejos de cualquier lectura romántica que se pueda realizar, nosotros consideramos que en el subsuelo de lo político se configuran espacios de resistencia, proyectos políticos alternativos, emancipadores, comunitarios de armonía y libertad, pero también sus más cruentos antagonismos. En el subsuelo de lo político, convicciones políticas de diversa índole (desde anarquistas, progresistas e izquierdistas hasta derechistas y fascistas) adquieren sentido y los actores que las representan se organizan y diseñan tácticas, estrategias, y acciones con las cuales irrumpen, se enfrentan y se presentan en la escena pública contemporánea.

El análisis del subsuelo de lo político propone un diálogo crítico y toma distancia de aquellas formas de entender la acción política desde un punto de vista escolástico que, para parafrasear a Villarreal Velásquez (2015; 2017), desde lejos y desde arriba, con temor y desconfianza, observa las nuevas dinámicas de (des) politización en la región y las convierte en un residuo de lógicas "paternalistas", "clientelares" y "populistas". En el mejor de los casos, este punto de vista los convierte en ejemplos de la "anti-política", "las democracias en ciernes e imperfectas" o "los Estados fallidos". Así, el subsuelo de lo político permite pensar de manera crítica la subordinación y sublevación como una relación dinámica y contingente en la que dominantes y dominados se enfrentan, tejen lazos y establecen acuerdos morales y políticos que, aunque frecuentemente no son perceptibles para los análisis filosóficos e institucionalistas, explican el cambio y la estabilidad. Como lo ha demostrado Rescher en otro lugar (2016 y 2018b), estas relaciones entre dominantes y dominados se convierten en una base integral e indispensable, aunque frecuentemente no reconocida, del sistema estatal institucionalizado.

Analizar el subsuelo de lo político también incluye las transformaciones tecnológicas, las nuevas herramientas de comunicación digital (Facebook, Twitter, WhatsApp, sitios web, portales, blogs, entre otros), sus usos e importancia para comprender su potencia en la acción política para hacer y deshacer vínculos, conflictos y negociaciones. Además abre una discusión sobre nuevas metodologías de la investigación que permitan abordar de mejor manera las temáticas mencionadas, que utilizan y combinan enfoques (sociológicos, antropológicos, comunicacionales, geográficos e historiográficos) tendientes hacia una decolonización de las formas tradicionales de producción del 
conocimiento sobre los fenómenos sociopolíticos, las experiencias colectivas y subjetivas que en ellos se forjan y se derruyen y que toman en cuenta el significado de la diversidad de las categorizaciones o posicionamientos sociales en los procesos de investigación (Rivera Garay, 2021).

Finalmente, esta perspectiva exige desentrañar las relaciones de poder entre los diversos actores presentes en el subsuelo político tomando en cuenta la construcción de la posición social de los sujetos políticos a partir de la combinación de varias características, como por ejemplo la adscripción étnica, la edad o el género (Segato, 2003; Federici, 2013), la colonialidad de las relaciones sociales y políticas (Rivera Cusicanqui, 2018; Mendoza, 2006); así como las categorizaciones étnicas combinadas con perspectivas de género (Yásnaya Aguilar, 2018 y 2019, Glady Tzul, 2014 y 2018, Aura Cumes, 2012 y 2014). Además, incorpora un enfoque que corresponde a un concepto latinoamericano de interseccionalidades como el desarrollado por Lélia Gonzalez (ver Ríos y Lima, 2020 y Buarque de Hollanda et al. 2020). ${ }^{5}$

\section{Sobre las contribuciones}

Los trabajos incluidos en este dossier (artículos y ensayos) discuten el subsuelo de lo político desde una amplia gama de momentos, discursos y prácticas, así como también enfoques teóricos y metodológicos para entender la complejidad inscrita en dicha categoría. Esta diversidad de campos, temas y metodologías se logró a partir del carácter abierto de la convocatoria y se refleja en la selección de los artículos.

Los procesos de movilización social y societal (que también incluye a los procesos electorales) sobre todo aquellos que han acontecido en países distintos como Chile, Argentina, Brasil, Ecuador, Colombia, y Guatemala se convierten en casos de análisis para comprender no solo la novedad de los mismos, sino también para explorar, debajo de la superficie, las distintas capas donde permanecen ocultos y sedimentados aquellos elementos que nutren gran parte de las potencialidades y los límites de estos procesos.

Desde una reflexión sobre las redes sociales digitales, Ana Tázia, Patricio de Melo Cardoso, Alexsandro Araújo Dantas y Patricia Gomes da Silva realizan un análisis sobre un grupo público de WhatsApp - Direita do Rio Grande do Norteque apoyó al candidato Jair Bolsonaro durante las elecciones de Brasil en 2018. Navegando en los turbulentos flujos de (des)información que se produjeron en la campaña electoral, los autores muestran de qué manera los partidarios del "bolsonarismo" convirtieron este "fenómeno político" en una forma de pós-

5 En este sentido, nuestro análisis comparte una visión más general sobre la persistencia de relaciones coloniales como lo exponen las y los pensadores decoloniales, sobre todo quienes analizan las relaciones de podery dominación del sistema mundial como, por ejemplo, Aníbal Quijano (2000) con su concepto de la colonialidad del poder y Pablo González Casanova (1965 y 2003) con su categoría de colonialismo interno. 
verdade y a su líder en un "mito". Un mito principalmente creado para combatir el miedo al comunismo y para satisfacer la necesidad de protección y seguridad de la población en aquel momento. En lugar de convertir a los seguidores de Bolsonaro en agentes portadores de cierta ingenuidad o ignorancia, el análisis propuesto en este ensayo resalta la eficacia de su actividad digital para crear nuevas formas y condiciones de enunciación de la verdad. De esta manera, para los autores, el "bolsonarismo" es el resultado del trabajo de una multiplicidad de actores-red y de una "experimentación tecnopolítica". Posts, memes, stickers, capturas de pantalla y audios circulados en el mencionado grupo de WhatsApp constituyen fuentes empíricas a través de la cuales los autores constatan cómo, por ejemplo, el hashtag \#BolsonaroTemRazao fue fabricado desde el subsuelo de lo político como una forma de mantener activos a los seguidores de Bolsonaro.

En una línea analítica similar, pero desde una orilla diferente, Elizabet Castillero-Ostio, Marta Gil-Ramírez y Antonio Castillo-Esparcia estudian la formación del activismo digital y del movimiento social durante la "Revolución de los Frijoleros" en Guatemala en 2020. Mediante un análisis de contenido y un análisis crítico del discurso, los autores constatan como las plataformas Facebook y Twitter se constituyeron en espacios de disidencia, articulación y organización de las acciones colectivas que dieron forma a la protesta social de octubre a diciembre de 2020. Haciendo usos de herramientas cuantitativas y cualitativas para comprender el uso estratégico de las redes sociales de cuatro organizaciones - Colectivo Estudiantil Universitario (CEU), Otra Guatemala Ya en Facebook, y los Landivarianos y el Movimiento Sindical, Indígena y Campesino Guatemalteco (MSICG) en Twitter-, los autores demuestran la capacidad que sus cuentas o perfiles tuvieron para, en un corto periodo de tiempo, atraer y, hasta cierto punto, comprometer a un número representativo de cibernautas que desarrollaron una conexión con el activismo social/digital y una afinidad hacia al proceso político que emergió en Guatemala. En suma, los autores demuestran cómo en el subsuelo de lo político no solo se crean posverdades sino también sensibilidades que alientan movimientos contestatarios como la denominada "Revolución de los Frijoleros".

Diosana Frigo en su artículo analiza la circulación de sentidos en el grupo de Facebook "Mulheres unidas pela Democracia Santa Maria (RS)" durante las elecciones presidenciales de Brasil en 2018. Las autoras realizan un análisis semiológico de los discursos y las interacciones que suscitan entre los miembros del grupo y desde ahí explican su adhesión al movimiento \#EleNão. De manera detallada y consistente, la autora demuestra como ciertos enunciados - "votar contra Bolsonaro", "posicionamento político" y "pedido de ajuda" - adquirieron un significado específico para las involucradas en el grupo mientras circulaban, eran replicados y comentados en la plataforma digital de Facebook. A pesar de que los esfuerzos realizados por este grupo no fueron suficientes para detener el avance electoral de Bolsonaro, Frigo destaca la capacidad de este movimiento para mantenerse activo más allá del momento electoral. La unidad, 
el fortalecimiento y la afirmación de las mujeres como seres políticos es lo que queda como sedimentos de lo político después de una comprometida acción colectiva que nació en el marco de una polarizada disputa política/electoral.

Explorando las dimensiones globales de la comunicación desde el subsuelo de lo político, Constanza Andrea Hormazábal Durand analiza el caso de la performance del colectivo feminista Las Tesis. Desde una perspectiva de la comunicación política discute el alcance global y la apropiación de esta performance analizando las bases de su repercusión. Su artículo estudia, sobre todo, el efecto que el movimiento tuvo en las redes sociales alrededor del mundo. La autora señala que las bases centrales para esta repercusión son su interpelación directa al sistema político (y podríamos decir a la sociedad en general), la unión clara entre teoría y praxis que se da en la calle como escenificación en un espacio público abierto a todes $\mathrm{y}$, finalmente, las vías de comunicación utilizadas tanto por los medios de comunicación más clásicos como también por las redes sociales, donde se multiplica la difusión de la performance de Las Tesis. En su conjunto, el artículo muestra que tanto las redes sociales como también la presencia en la calle, son dimensiones integradas al subsuelo de lo político.

De una manera similar, Janara Sousa y Gerson Scheidweiler se enfocan en el campo de la comunicación con relación a cuestiones de género y más específicamente en la importancia de los derechos a la comunicación para la visibilidad de discursos y exigencias feministas. Con base en un trabajo bibliográfico, los autores critican al concepto de esfera pública de Habermas y constatan que existen distintos públicos con diversas condiciones, contextos, entre otros. Estos públicos, señalan los autores, son muy diversos en cuanto a su alcance e influencia discursiva. Tomando como ejemplo los procesos participativos en las conferencias mundiales de mujeres organizadas por la ONU, argumentan que el derecho a la comunicación es un mecanismo de resistencia central. Por medio de éste se busca lograr que se amplíe la visibilidad de diversas voces de mujeres en las políticas públicas. No obstante, los autores constatan que finalmente es difícil llegar a un cambio real y más amplio, a pesar de tener acceso a espacios de comunicación. Por ello sugieren como solución la transferencia de voces de mujeres con diversos trasfondos a espacios formales de política y decisión. Transferencia que debe ser apoyada por el derecho a la comunicación para así dejar de ser un "contrapúblico subalterno". Al final, el artículo hace evidente el problema de la relación entre el subsuelo de lo político y los espacios formales.

En su trabajo Ana Karen Grünig realiza un análisis (hermenéuticointerpretativo) de la política de identidad narrativa (individual y colectiva) a partir de la producción audiovisual que se realizó como parte del Plan Operativo de Fomento y Promoción de Contenidos Audiovisuales Digitales del SATVD-T en Argentina entre los años 2010 y 2015. Particularmente, la autora centra su atención en el género de ficción. Su estudio indaga las condiciones de producción 
y las formas de interpretar mitos, leyendas y otros tipos de "creencias populares" que surgieron dentro de las narrativas de las series de ficción. Grünig demuestra cómo el uso de los mitos populares (desde las regiones federales) puede servir para visibilizar historias y experiencias que generalmente han permanecido ocultas (por la televisión nacional). Más importante que aquello, la autora señala como estas mitologías, so pena de las limitaciones y contradicciones inherentes a su producción, disputan la configuración de nuevos lugares, formas de enunciación (abordajes narrativos y estéticos) e identidades dentro del orden del discurso oficial/hegemónico. El trabajo de Grünig, en resumen, da cuenta de las operaciones políticas que se ponen en escena dentro de un "territorio audiovisual" en disputa.

El caso de dos consultas populares contra proyectos de extracción mineroenergética en los municipios colombianos de Arbeláez (Cundinamarca) y de Fusagasugá (Cundinamarca) son el punto de partida desde el cual Marisabel García Acelas y Robert Adrian Quintero Leguizamon, analizan en su artículo las prácticas de comunicación en el subsuelo de lo político desde una investigación empírico-participativa. Dichas prácticas cuentan con un repertorio amplio, incluyendo nanomedios y artes populares y se vinculan con acciones de organizaciones sociales que promovieron la consulta, como paros y marchas, pero también luchas más generales por la producción agroecológica, la defensa del territorio y la reivindicación del campesinado como un sujeto de derechos. Además, se reconoce públicamente el conflicto socioambiental, la opinión pública se diversifica y se permite el establecimiento de formas de participación popular. El artículo muestra de qué manera las consultas funcionan como instrumentos de defensa de autonomía, autogestión y cuestionamiento al modelo de desarrollo. Esta campaña convocó a actores sociales heterogéneos y "con la intención de participar en asuntos públicos, detonaron potentes ejercicios comunicativos de carácter contrahegemónico". Los autores observan como esto se logró a través de una "herramienta de trabajo popular" y la formación de un "ethos cultural local" que integra a nuevos miembros desde la práctica y logra así, crear una comunicación desde abajo.

En su contribución, Andrés Lozano Reyes se enfoca en los procesos de comunalidad que encuentran espacio en el subsuelo de lo político. Analizando la gobernabilidad comunal campesina en el caso empírico de la Asociación Campesina del Catatumbo (Colombia), el autor estudia las formas de gobierno en el subsuelo de lo político que están dirigidas hacia lo común. Su investigación desglosa los mecanismos y las lógicas de gobernanza campesina. Estas dinámicas están marcadas por la diversidad, por lo que el autor hace especial énfasis en que es indispensable reconocer la multiplicidad de formas que caracterizan al subsuelo de lo político. De esta manera, Lozano Reyes propone un acercamiento metodológico con categorías intermedias tomando en cuenta la relación entre espacios societales diversos y un Estado que invisibiliza a la heterogeneidad social. El autor señala la importancia "de tener en cuenta las 
especificidades de las formaciones sociales locales, a la vez que se piensan las regularidades continentales en un sentido de síntesis". El artículo analiza con especificidad la política y el trabajo como elementos centrales y reguladores de formas de vida comunitaria en América Latina. En el caso de Catatumbo, por ejemplo, se observan altos grados de autonomía en la reproducción de lo común, los cuales el Estado colombiano no ha podido debilitar completamente, gracias a la diversidad presente. En conjunto, el artículo de Lozano Reyes muestra como la reconstrucción local de alternativas desde el subsuelo político son posibles y factibles.

Lucas Milhomens, de manera similar, enfatiza la importancia de la diversidad de alianzas en el subsuelo de lo político, así como también del uso de múltiples formas de acción para lograr ser visibilizados en espacios formales. El autor estudia dos casos de organizaciones indígenas en Brasil, protagonistas transversales en varias luchas importantes presentes tanto en noticias de medios masivos como en espacios de comunicación alternativa. Su presencia, según argumenta el autor, se consiguió a partir de la construcción de amplias redes de apoyo y de movilización con un carácter de comunicación anticolonial. Lo que, entre otros, les ha permitido también alcanzar gran visibilidad mundial y nacional. Esto es esencial, ya que, además de los grandes desafíos históricos que enfrentan estas organizaciones, la situación es más difícil en estos años. El gobierno actual lidera una guerra ideológica que, aparte del desmantelamiento de servicios estatales, sitúa a los territorios indígenas en una condición de riesgo y favorece a proyectos neoliberales. Milhomens demuestra cómo las organizaciones indígenas se convierten en el mayor opositor a la gestión del gobierno federal. Sus redes representan una compleja articulación de varios movimientos sociales, grupos políticos y otros actores. Redes que son construidas tanto en espacios virtuales como presenciales y forman la base para iniciar varias actividades, procesos legales, movilizaciones, campañas, entre otros. Estas redes, como se observa en el artículo, además, comparten un elemento importante: la apropiación de nuevas formas comunicacionales, lenguas inherentes al medio virtual y un ejercicio reflexivo de sus propias limitaciones.

Por último, llegar a los sustratos del subsuelo de lo político, también implica el desarrollo de nuevas metodologías. En su ensayo, Lorena Marín Gutiérrez presenta una propuesta metodológica para sistematizar y analizar las diversas y complejas dinámicas de acción colectiva que emergieron durante el Paro Nacional en la ciudad de Cali-Colombia entre abril y junio de 2021. La "espectrónica", como lo denomina la autora, es una apuesta metodológica que, inspirada por algunos supuestos epistemológicos de la educación popular, sirve para explorar y leer, desde abajo, la complejidad de las múltiples voces que dieron forma a la acción colectiva; así como también los sentidos (educativos), lazos de solidaridad y horizontes emancipatorios que se inscribieron en las 
protestas de abril y que siguen presentes en la memoria de quienes participaron activamente en ellas.

\section{Referencias bibliográficas}

Aguilar, Y. (2019). Mujeres indígenas, fiesta y participación política, Dossier Feminismos, Revista de la Universidad de México, UNAM, 854, 33-39.

Aguilar, Y. (2018). La sangre, la lengua y el apellido. Mujeres indígenas y estados nacionales. En Jauregui, G., \& Abenshushan, V. (Ed.), Tsunami (pp.25-40). México: Sexto Piso.

Bringel, B. \& Pleyers, G. (Eds.). (2017). Protestas e Indignación Global. Los Movimientos Sociales En El Nuevo Orden Mundial. Buenos Aires: Consejo Latinoamericano de Ciencias Sociales.

Büschges, C. \& Pfaff-Czarnecka, J. (2003). La etnización de lo político. Etnicidad, Estado-Nación y Globalización en la Sudamérica Andina y en Asia del Sur. Colombia y América Latina: procesos de globalización, gobernabilidad, ambiente e interculturalidad., CD-ROM, p.14. Popayán: Universidad del Cauca.

Carneiro, S., de Hollanda, H. B., Gonzalez, L. \& Nascimento, B. (2020). Interseccionalidades: pioneiras do feminismo negro brasileiro. Brasilien: Bazar do Tempo.

Castells, M. (1996). La Era de La Información: Economía Sociedad y Cultura, La Sociedad Red, 1. México: Siglo XXI.

- - - (2012). Redes de Indignación y Esperanza. Madrid: Alianza Editorial.

Cumes, A. (2014). La "india" como "sirvienta": Servidumbre doméstica, colonialismo y patriarcado en Guatemala (Tésis de Doctorado). México: CIESAS.

Cumes, A. (2012). Mujeres indígenas patriarcado y colonialismo: un desafío a la segregación comprensiva de las formas de dominio. Anuario Hojas de Warmi, 17.

Federici, S. (2013). Revolución En Punto Cero. Trabajodoméstico, Reproducción y Luchas Feministas. Madrid: Traficantes de sueños.

Gerharz, E. \& Rescher, G. (2020). Global South. En Hollstein, B. et al. (Eds.), Soziologie: The State of Sociology in the German-speaking World, Special Issue Soziologische Revue (pp. 165-181). Oldemburgo: de Gruyter.

González Casanova, P. (1965). Internal Colonialism and National Development. Studies in Comparative International Development, 1(4), 27-37.

González Casanova, P. (2003). Colonialismo interno (una redefinición). México: UNAM.

Gonzalez, L. (2020). Por um feminismo afro-latino-americano. Rio de Janeiro: Zahar.

Lachenmann, G. \& Dannecker, P. (2008). Negotiating Development in Muslim Societies: Gendered Spaces and Translocal Connections. Lanham: Lexington Books.

Latour, B. (2007). Nunca fuimos modernos: ensayo de antropología simétrica. Buenos Aires: Siglo Veintiuno Editores.

Marchart, O. (2009). El Pensamiento Político Posfundacional. La Diferencia Política En Nancy, Lefort, Badiou y Laclau. Buenos Aires: Fondo de Cultura Económica Argentina.

Mendoza, B. (2006). Los Fundamentos no Democraticos de la Democracía: un enunciado desde Latinoamérica pos occidental, Revista Centroamericana de Ciencias Sociales ( RCCS ), 3(2), 85-94.

Mouffe, C. (2007). En Torno a Lo Político. Buenos Aires: Fondo de Cultura Económica Argentina.

Nageeb, S. (2007). Appropriating the Mosque: Women's Religious Groups in Khartoum. Afrika Spectrum, 42(1), 5-27. 
Quijano, Á. (2000). Colonialidad del poder, eurocentrismo y América Latina. Argentinien: CLACSO.

Rescher, G. (2018)a. Doing Democracy in indigenen Gemeinschaften: Politischer Wandel in Zentralmexiko zwischen Transnationalität und Lokalität. Bielefeld: Transcript.

Rescher, G. (2018)b. Indigenous People and Local Political Change in Contemporary Mexico: Dimensions of Indigeneity as Political Ressource. En Gerharz, E., Uddin, N., Chakkarath, P. (Ed.), Futures of Indigeneity: Spatiality, Identity Politics and Belonging (pp.Zahl - Zahl). New York/ Oxford: Berghahn.

Rescher, G. (2016). Transnationale indigene Migration als Element der Herstellung von Demokratisierungsperspektiven in Zentralamerika. En Eigenmann, P., Geisen, T. \& Studer,T. (Eds.), Migration und Minderheiten in der Demokratie: Politische Formen und soziale Grundlagen von Partizipation (pp. 153-173). Wiesbaden: Springer VS.

Rivera Garay, M. G. (2021): El uso de la etnografía multisituada para el estudio de una comunidad indígena que práctica la migración transnacional a los Estados Unidos. En González Ramírez, J. (Ed.), Geografías de la Diferencia: Irrupciones de las desigualdades en los procesos de la globalización (pp. 167-192). Ort: Verlag. vllt Puebla: BUAP.

Rivera Cusicanqui, S. (2018). Un Mundo Ch'ixi Es Posible. Ensayos Desde Un Presente En Crisis. Buenos Aires: Tinta Limón.

Scott, J. C. (1990). Domination and the Arts of Resistance: Hidden Transcripts. New Haven/ London: Yale University Press.

Scott, J. C. (2009). The Art of not being Governed. New Haven/ London: Yale University Press.

Segato, R. (2003). Las Estructuras Elementales de La Violencia. Ensayos Sobre Género Entre La Antropología, El Psicoanálisis y Los Derechos Humanos. Buenos Aires: Prometeo-Universidad Nacional de Quilmes.

Tapia, L. (2008). Política Salvaje. La Paz, Bolivia: Muela del Diablo, Comuna, Consejo Latinoamericano de Ciencias Sociales.

Torret, J. (2013). Tecnopolítica: la potencia de las multitudes conectadas. El sistema red 15M, un nuevo paradigma de la política distribuida. Barcelona: Internet Interdisciplinary Institute, Universitat Oberta de Catalunya.

Tzul, G. (2018). Sistemas de gobierno comunal indígena: la organización de la reproducción de la vida. En Meneses, M. P. y Bidaseca, K. A. (coord.), Epistemologías del sur (pp.385397). Buenos Aires: CLACSO.

Tzul, G. (2014). Las luchas de las mujeres indígenas en Chuimek'ena', Guatemala: Una aproximación teórica a las estrategias. Contrapunto 5: Feminismos. La lucha dentro de la lucha, 65-75.

Villarreal Velásquez, J. (2015). La Intermediación Como Práctica Sociopolítica de Los Sectores Urbano Marginales de Guayaquil En El Contexto de La Revolución Ciudadana (Tesis de Maestría). Quito: Facultad Latinoamericana de Ciencias Sociales FLACSO Ecuador.

-_- (2017). The 'Art of Service': Intermediation as a Sociopolitical Practice of the Urban Poor in Guayaquil. En Rehm, L., Kemner, J. \& Kaltmeier, O. (Eds.). Politics of Entanglement in the Americas. Connecting Transnational Flows and Local Perspectives, 19 (pp.155-70). Bielefeld: WVT Wissenschaftlicher Verlag Tier.

Vincent, J. (2002). The Anthropology of Politics: A Reader in Ethnography, Theory, and Critique. Malden: Wiley-Blackwell.

Nugent, D. \& Vincent, J. (2004). A Companion to the Anthropology of Politics. Malden: Wiley-Blackwell. 